\title{
Construction Project Monitoring with Site Photographs and 4D Project Models
}

DOI 10.5592/otmcj.2014.3.5 Research paper

\section{Keywords}

\author{
Miroslaw J. Skibniewski \\ Dept. of Civil \& Environmental Engineering \\ A.J. Clark School of Engineering; \\ University of Maryland College Park \\ mirek@umd.edu
}

EARLY AND ACCURATE MEASUREMENT OF PROGRESS IS CRUCIAL FOR THE SUCCESS OF A PROJECT. Early detection of discrepancies and deviation from the as-planned data allow more time for project participants to identify the best solution. Monitoring the construction progress requires the Project Managers to analyze a lot of as-built data. This time-consuming process is prone to error. To overcome this problem, people have started adopting Building Information Models (BIM) for monitoring the construction progress. In this paper, we have discussed how the site photographs, which are readily available at all project sites, can be effectively used for monitoring the progress. The $4 \mathrm{D}$ as-planned model is prepared at the beginning of the project. Then, a 3D model is reconstructed from time-lapsed as-built photos. For any given time, the progress can be tracked by comparing the $4 \mathrm{D}$ model and the $3 \mathrm{D}$ as-built model. The progress data is extracted by superimposing the reconstructed scene over the $4 \mathrm{D}$ as-planned model. The reconstructed model allows the as-built photographs to be geo-registered by the $4 \mathrm{D}$ as-planned model. As a result of this comparison, the progress data is then visualized in an AR environment. Progress is visualized in the $4 \mathrm{D}$ model using a quadrangle visualization scheme. This type of approach presents a realistic understanding of the construction progress and is a very good communication tool between project participants. We have discussed both interior and exterior construction progress monitoring is this paper 


\section{INTRODUCTION}

The traditional method of progress monitoring requires field engineers to collect data, send it to the office and develop progress reports through this large amount of data. Office staff needs to extract the data from different construction documents, and producing the reports is extremely time-consuming for them; yet, it still does not communicate the project progress quickly and effectively (Peña-Mora et al., 2009). Project managers and superintendents require more time to resolve rather than identify the issues. With continually increasing complexity of projects, progress monitoring methods are also changing. The construction industry is replacing the traditional monitoring methods by automated solutions to accomplish this task. $2 \mathrm{D}$ drawings are being replaced by 3D CAD files and Building Information Models (BIM). These models provide comprehensive information about the building's design, and their use has been rapidly increasing in the construction industry. There has been a large body of research on automatic tracking of construction progress. Most of these techniques measure the physical quantities of work performed by using spatial sensing technology (Turkan et al., 2012). Using laser scanners for producing an as-built $3 \mathrm{D}$ model of the building and comparing it with the $4 \mathrm{D}$ model is an effective technique for monitoring progress. However, laser scanners are expensive and thus not a good resource for small-scale projects. In this paper, monitoring progress is proposed by overlaying site photographs on $4 \mathrm{D}$ asplanned models such as those initially suggested by Peña-Mora et al. (2009). In this technique, a $3 \mathrm{D}$ as-built model is prepared using time-lapsed photographs and this model is superimposed on the $4 \mathrm{D}$ as-planned model to detect progress deviations. It is easier to track the progress by looking at this visual imagery than by an analysis of scheduling charts, progress curves, and other forms of project reporting measures.

\section{Monitoring of construction progress}

A $4 \mathrm{D}$ model is a $3 \mathrm{D}$ model with a time component linked with it. A $4 \mathrm{D}$ model is developed by first preparing a $3 \mathrm{D}$ model and a construction CPM schedule separately. The $3 \mathrm{D}$ model is then separated into appropriate construction assemblies, and these components are then linked to the corresponding activities in the CPM schedule (Lingyun 2007). The $4 \mathrm{D}$ model provides a depiction of what the construction project site will look like on any specific date. There are a number of commercially available software solutions available for preparation of $4 \mathrm{D}$ models. In this paper, progress monitoring is discussed of exterior and interior construction in two different sections, because the practical approach to monitoring progress is somewhat different in both cases.

\section{Exterior Construction Progress monitoring}

Progress of a construction project can be tracked by looking at the deviation in as-built and as-planned models. Golparvar-Fard et al. (2009) introduced an idea of superimposing a $4 \mathrm{D}$ asplanned model with site photographs for representing progress deviations in an AR environment. An augmented reality (AR) environment combines real and virtual worlds for better visualization of the progress. The two models - real and virtual world - that are compared can be explained as follows.

4D as-planned model (virtual world) Preparing a $4 \mathrm{D}$ model of a project in the planning stage gives greater predictability, lowers cost, saves time and reduces waste. The owners have a better visualization of their project. Any discrepancies or clashes can be detected well in advance, and alternatives can be explored well in advance. This saves a good amount of money and time. The $4 \mathrm{D}$ as-planned model serves as a progress monitoring tool.
3D reconstructed model by site

photographs (real world)

The next step for tracking progress is to develop a $3 \mathrm{D}$ model using construction site photographs. It is really difficult to reconstruct a model by photos that keep on changing throughout the day due to progress and continuous movement of machinery at the construction site. The final color-coded AR imageries are a product of a series of modules. The process of visualization can be explained below.

As shown in Fig. 1, the process consists of collecting, analyzing and recording of data. The data is collected by two sources, i.e., the as-planned model and site photographs. The $3 \mathrm{D}$ as-planned model (in an Industry Foundation Classes (IFC) format) and the site photographs represent the "product model." The as-planned schedule and as-built schedule represent the "process model." The "cost module" is represented by the estimated construction cost and the actual construction cost (Peña-Mora et al., 2009). In the analysis stage, the product model and process model are combined to produce a $4 \mathrm{D}$ as-planned model and time-lapse photographs. The time-lapse photographs are then used to reconstruct an as-built $3 \mathrm{D}$ model. The process of reconstructing this $3 \mathrm{D}$ model using SfM technique is explained in a separate section. The construction progress at any given time is then monitored by superimposing the $4 \mathrm{D}$ as-planned model over the $3 \mathrm{D}$ reconstructed model. The deviations in the models can then be detected either manually or automatically. Any deviation in the cost can also be determined at this stage by comparing the funding spent with the funding allocated for the work. This cost information helps in deriving the earned value analysis (EVA). EVA is important for arriving at a status information of the construction progress. Progress of a project is not conveyed by simply determining whether it is ahead or behind the schedule. Cost performance is measured by 


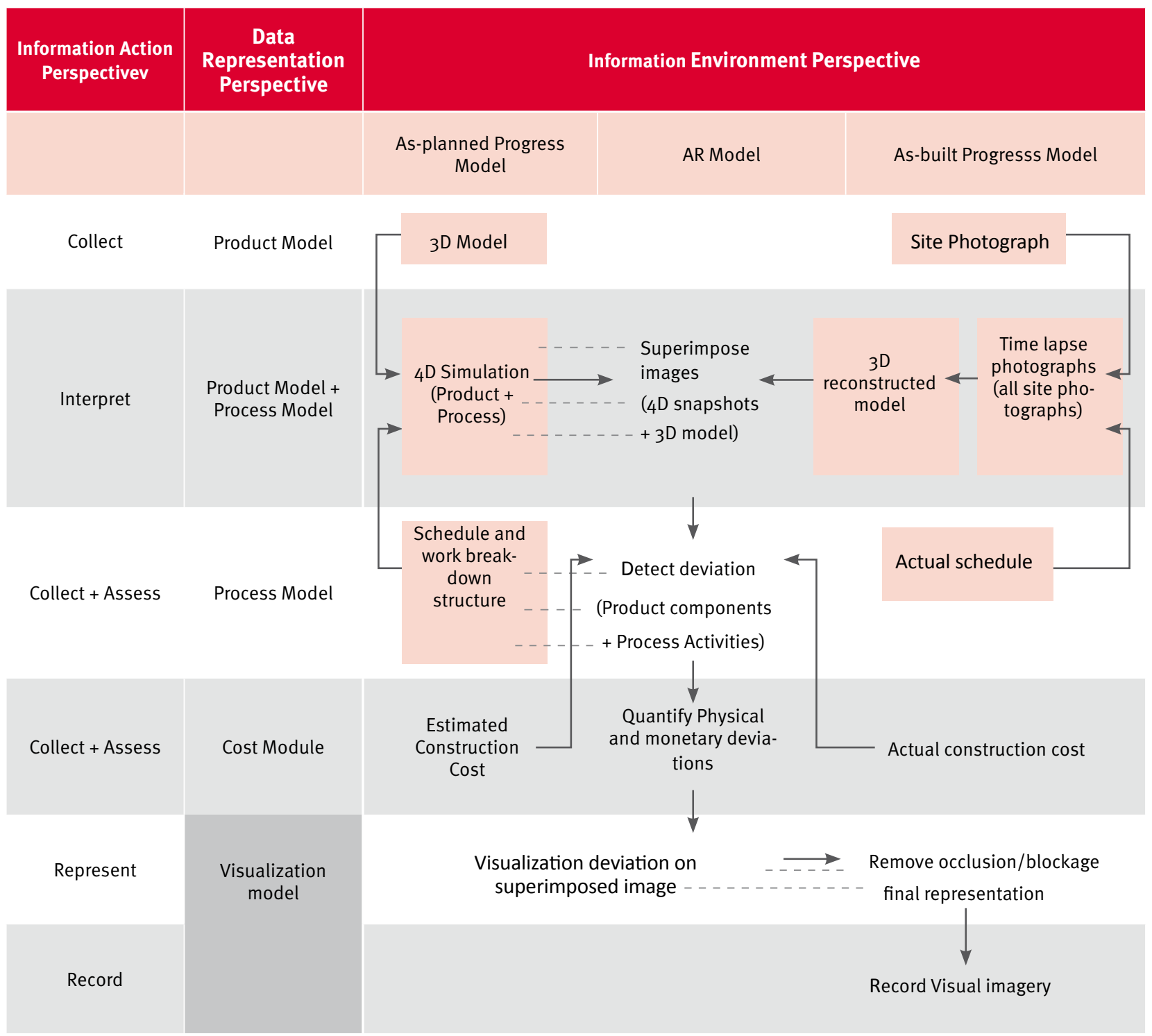

A.

B.

...C

Figure 1 Elements of the visualization process of construction project monitoring task.

comparing the actual cost to accomplish a task with the cost planned for that task. The 3D as-planned model, schedule and work breakdown structure are taken as baseline measurements for monitoring the progress. Figure 1

A detailed schedule of the construction activities should be prepared for correct progress measurements because the $4 \mathrm{D}$ as-planned model is prepared according to the schedule and work-breakdown structure. The 4D asplanned model visualizes all the activities mentioned in the schedule (except the non-physical activities). After the deviations in the physical activities and cost are detected and quantified, they have to be visualized (Peña-Mora et al., 2009). For visualizing the progress accurately, the as-planned model is color-coded and superimposed on the site photograph. The visualization technique is described in a subsequent section in this paper. Although a quadrangle color scheme has been proposed, the image in Fig. 2 shows only green and red colors. The green color represents the activities that are on time and on budget, the red color represents activities that are behind schedule and over budget. Any occlusion or blockage that is caused by superimposing the image is removed at the end. Figure 2

\section{SfM technique for reconstructing 3D model}

For reconstruction of the as-built scene, the technique called Structure from Motion (SfM) can be used. For reconstructing the scene automatically, one needs to know the relative 


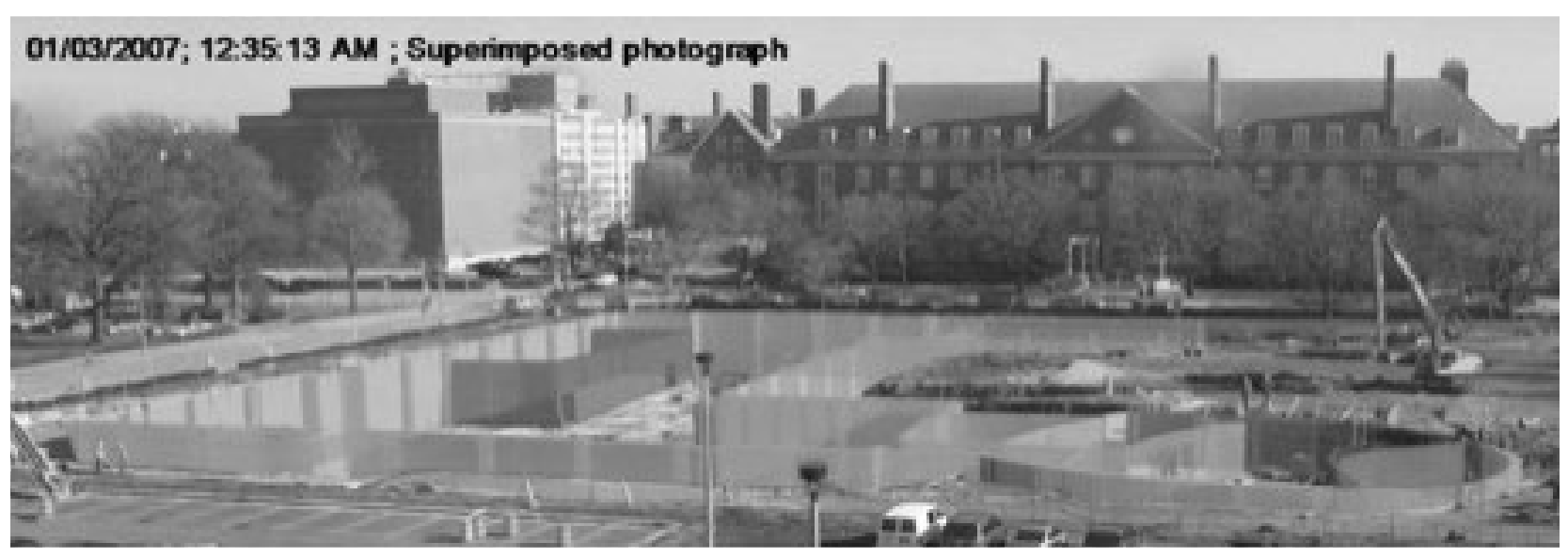

Figure 2 3D as-planned color coded model superimposed on the site photograph.

(Source: Golparvar-Fard et al. [2], 2009)

locations, orientation, zoom or focal length of the photograph. The process of reconstruction consists of four steps (Golparvar-Fard et al., 2009):

1. Detecting features in all the images

2. Matching these features across image pairs

3. Combining the matches into correspondence tracks across several photos

4. Estimating the geometry of the scene and location of the cameras using these correspondences

The first step is to detect distinct features in each image that could be used to prepare the initial arrangement of the scene. In this paper, we will discuss the SIFT (Scale Invariant Feature Transforms) key point detector (Lowe 2004). This technique has showed good invariance to image transformations in the experiments performed by (Mikolajczyk et al., 2005). There are thousands of SIFT key points in a typical image. After detecting the key points, we matched key point descriptors in an image pair. Once a set of geometrically consistent matches is found in an image pair, the matches are organized into tracks. A set of connected matching key points across several images is called tracks. The extrinsic (translation, rotation) and intrinsic properties (focal length, distortion) of the camera and $3 \mathrm{D}$ location for each track are recovered at this stage. Initially, this is done just for our first image pair, because it is important to get good initial estimates. For estimating the parameters of all tracks and cameras, we follow an incremental approach. For robustly estimating the two-frame reconstruction, the initial pair should have a large amount of matches. For this purpose, we choose an image pairs, which have a maximum number of matches (at least 100) to be the initial pair. Nister's five-point algorithm (Nister 2004) is used for estimating the extrinsic parameters of the camera, and then the tracks visible in this image pair are triangulated. After this, another camera is added to the optimization. Direct linear transform (DLT) technique is used for finding the new camera's extrinsic parameters. The intrinsic parameters of the new camera are estimated with the help of DLT technique and EXIF-Exchange image file format-tags of JPEG images. After this, the bundle adjustment algorithm is run, which allows only the new camera and points it observes to change keeping the rest of the model constant. Finally, the points observed by the new camera are added to the optimization algorithm. The points are added only when it is observed by at least one of the already recovered cameras and if a well-conditioned estimate of its physical location can be made by triangulating the point. All the pairs of rays that could be used for triangulating that point are considered for estimating the location. The angle of separation between these pair of rays should be more than threshold limit (2.0 in this case), for the point to be triangulated. After the addition of all the points, the global bundle adjustment is run to refine and reconstruct the entire scene. This procedure is continued in all of the cameras. The data structure used for visualizing the as-built reconstructed scene is as follows (GolparvarFard et. al, 2009):

a. A set of key points (with its 3D location and an average color determined by the site images from which this key point has been observed)

b. A set of camera (with known extrinsic and extrinsic parameters)

c. A mapping between key point and all the cameras that observe that key point

Along with this information the "number of cameras" observing that point, "local coordinates" of the point in the image and "SIFT key point index" is also stored for all the key points.

Figure 3

For providing better resolution of the reconstructed scene, the camera frustum is texture mapped. This allows the user to zoom in and get the information 

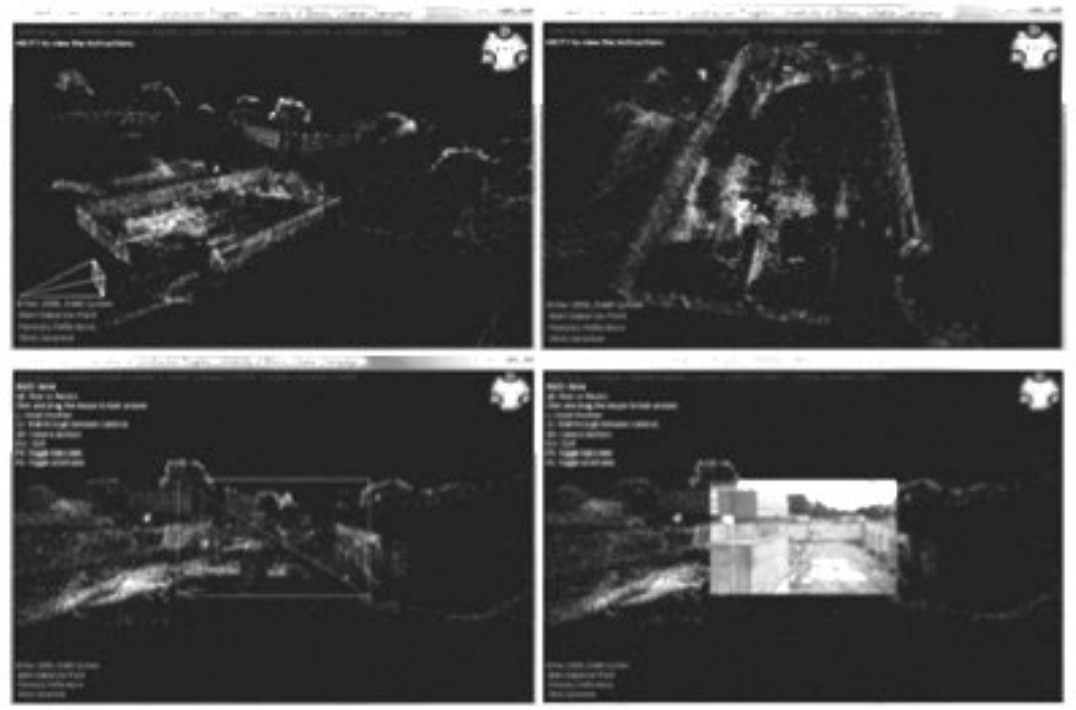

Figure 3 A sparsely reconstructed scene of the project site (Source: GolparvarFard et al. [1], 2009) For providing better resolution of the reconstructed scene, the camera frustum is texture mapped. This allows the user to zoom in and get the information about the productivity, construction progress from the reconstructed scene (Golparvar-Fard et al., 2009)

about the productivity, construction progress from the reconstructed scene (Golparvar-Fard et al., 2009)

\section{Geo-registration}

Geo-registration is the process of aligning coordinates of one image/ model to image/model with known coordinates. By the SfM technique, we get the relative position of the camera. We use the geo-registration process to determine the exact camera coordinates. This is done by aligning the as-built reconstructed model with the as-planned model. We need the exact coordinates of the as-built scene to get it aligned with the as-planned model. Theoretically, the estimated coordinates of the reconstructed scene are related to the absolute coordinates (exact location) by a rotation, uniform scale transformation and global translation (Snavely et al., 2007). For correctly determining the transformation, the sum of squared residual errors between the model and set has to be minimized. This is done by registering a set of as-built 3D model points to asplanned $3 \mathrm{D}$ set of points. The process of specifying the set of correspondences between the $3 \mathrm{D}$ as-planned and points in an image can be done manually (Golparvar-Fard et al., 2009). The same registration will be held for all the images. The geo-registration process allows the as-built site images to exactly superimpose on the $4 \mathrm{D}$ asplanned model.

\section{Interior construction progress monitoring}

The schedule for interior work is not as detailed as it should be for proper tracking of the progress of interior construction. There is a significant schedule discrepancy in the interior construction, which makes it difficult for project managers to predict accurate subcontractor's progress and productivity (Seungjun et al., 2011). Many cost overruns and schedule delays are caused, due to the limited visual understanding, manually intensive process and complicated nature of the interior construction progress. Thus, the interior construction progress reports should have object-oriented aspects and visual data for in-depth progress information. In this paper, we will discuss the object-based project monitoring approach. In this approach, the as-built construction photographs are compared with the as-planned project goals. The object-based model with BIM is used to represent geometric information of construction components into meaning object. This is done through an automated analysis using computer vision techniques. The interior construction progress monitoring using BIM can be explained as follows.

\section{Decomposition of interior} construction objects

For creating a relationship to measure the interior construction progress, we need that level of detailed objects and activities to be the same as defined in the as-planned model. The construction schedule for interior construction is not as detailed as the exterior construction schedule. For effective interior construction progress monitoring, the interior construction processes should be described in more detail. As can be seen in Figure 4, HVAC ductwork in ceiling spaces is scheduled for 60 days. It is not possible in this case to monitor the progress on a weekly basis. The activities should be treated as objects, so that it can be represented in a $3 \mathrm{D}$ model according to the percentage of work done. The activities can be divided down further on the basis of location information, e.g., zone or floor in the building. Thus, for representing all interior construction progress, we need to detect objects and location information of their activities. For instance, there are four HVAC duct units to be installed on second floor and 3 have been installed already; then, the percentage of progress can be calculated. This progress can then be compared by the as-planned model and then be represented on the ${ }_{3} \mathrm{D}$ model by a using color-coding metaphor. By preparing a detailed schedule, the efficiency of interior construction is improved because the deviations in 


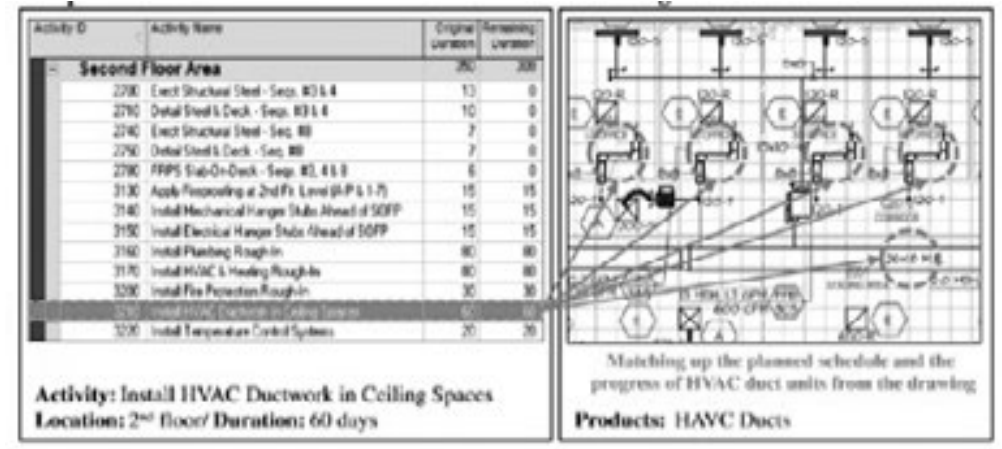

Figure 4 Relationship between product's schedule and the product (Source: Seungjun et al., 2011).

as-built and as-planned model can be assessed quickly.

For monitoring the progress of nonphysical activities of interior construction (e.g., installing water pipes) should be controlled and monitored by using detailed physical objects from the $3 D$ model (Seungjun et al., 2011).

\section{Conceptual model for interior construction progress monitoring using BIM}

Besides showing $3 \mathrm{D}$ geometric data and material properties, BIM also includes schedule, cost, safety information, equipment warranties, product data sheets, etc. BIM facilitates automatically track the construction progress according to the as-planned schedule for interior components. In actual conditions, the construction progress is not adjusted exactly according to the work being done because the schedule does not exactly describe the activity that is being performed. For example, in Fig. 6, installation of a duct on the second floor takes 60 days, and this is represented in the schedule only by one activity, but in actuality there are many building components included for this work. With the use of BIM, these HVAC duct elements are considered and a better visualization of interior construction progress is achieved.

Figure 5 shows how the interior construction progress is monitored using as-built construction photographs and BIM. The as-planned BIM is prepared by monitoring thousands of construction elements. The interior construction elements are decomposed from the as-built photographs. Only the elements that can be seen in the asbuilt photographs have to be taken in account in the as planned model and their schedules for monitoring the progress. For example, in Fig. 5 one only considers drywall components for calculating the construction progress because it is only visible in the as-built photographs. First of all, the as-built construction elements whose progress needs to be monitored are decomposed. This is done by using the as-built photographs. After decomposition, these components are selected in Industry Foundation Classes (IFC $2 X_{3}$ ) format for matching with the as-planned model. IFC is a file format that enables interoperability in AEC industry (Renauld et al., 2008). This file format is commonly used for BIM. Here, this format allows the data to be exchanged between the as-built photographs and the as-planned model. Comparing the as-built data with the as-planned data provides the progress of the construction. Deviations in the schedule and cost are detected by comparing the actual data with the as-planned data. This data can be used for measuring the percentage of work done. This information and cost data can then be used to measure Actual Cost, Planned Value and Earned Value. CPI and SPI can then be calculated by these values. This information is then used to color code the $4 \mathrm{D}$ as-planned model either manually or automatically. Fig. 6 shows the as-built photo superimposed on color coded as-planned model of interior construction. The green color represents the activities ahead of schedule and red represents the activities that are behind schedule.

\section{Visualization of construction progress}

Proper visualization is very important for tracking the project's progress. By just representing whether the project is ahead of schedule or behind the schedule does not really convey the progress. The project can be ahead of schedule, but could have a lot more money spent than planned. The concept of EVM solves this problem. EVM is a project management technique that accurately conveys the project performance. It combines the measurement of scope, schedule and cost. By the

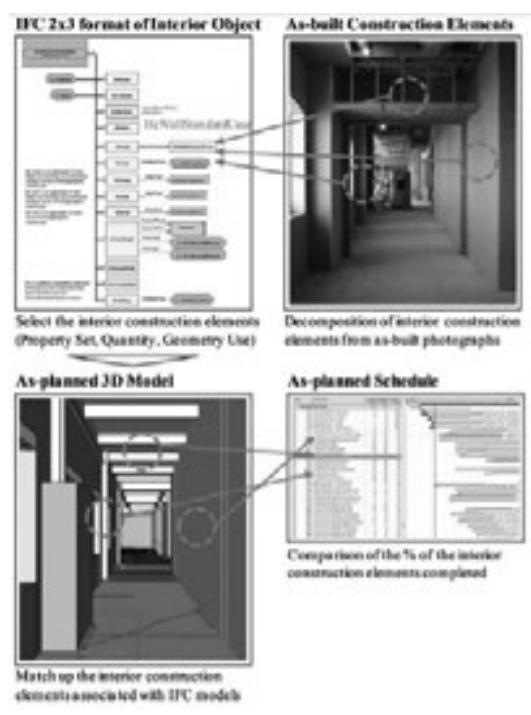

Figure 5 Using BIM for interior construction progress monitoring by as-built photographs

(Source: Seungjun et al., 2011). 

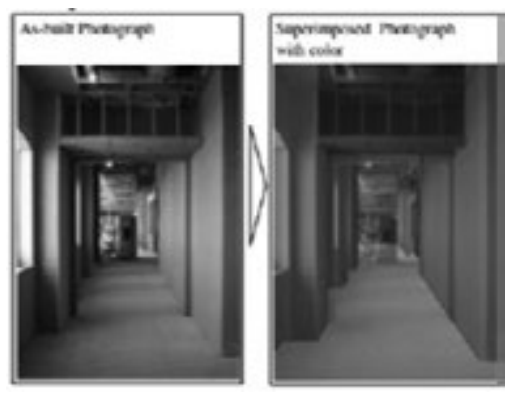

Figure 6 Colored representation of the interior construction progress. (Source: Seungjun et al., 2011).

earned value analysis method, progress can be monitored and also the future performance can be predicted. One of the challenges with EVM is to represent all the information in one view. However, Song et al., (2005) proposed an idea for representing the progress and critical information of the activities by using a color-coded scheme. They proposed an idea of representing different data sets on border, faces of the geometric $3 \mathrm{D}$ model and also by applying dynamic movements such as rippling and shaking of the $3 \mathrm{D}$ model. However, in this paper, we will not describe about representation by applying the dynamic movements.

It is the responsibility of project manager to produce useful information from the raw data collected from the field. In order to overcome and

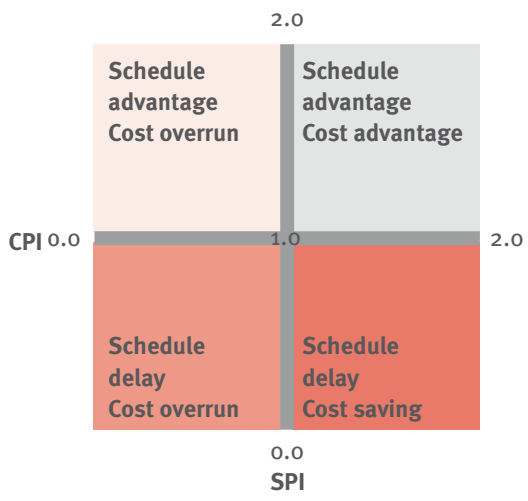

Figure 7 Quadrangle scheme for visualizing progress

(Song et al., 2005) minimize future problems, the project manager should analyze the data and forecast the future conditions. There are three types of information that are important for project managers to properly monitor the progress (Song et al., 2005).

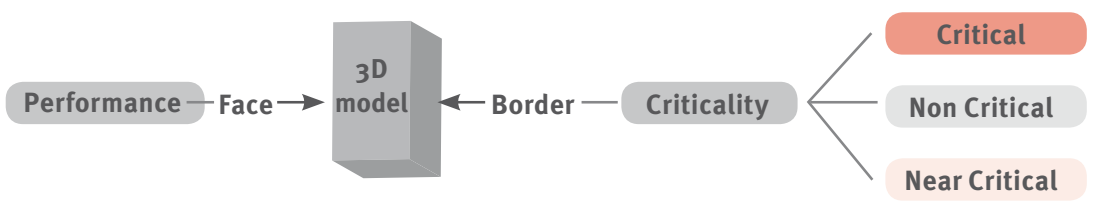

Figure 8 Representation of criticality and performance information of the project on a 3D model (Song et al., 2005)

1. Performance indexes:

CPI/SPI- CPI and SPI are tools for conveying the current progress and predicting the future progress of a project. These are represented simultaneously using a quadrangle color scheme (Fig. 7).

The performance indices are represented on the face of the $3 \mathrm{D}$ model. The green color represents perfect performance, blue represents that the project is ahead in schedule (SPI) and under budget (CPI), orange indicates a project is behind schedule and under budget, yellow indicates ahead in schedule but over budget and red indicates that the project is behind schedule and over budget. There is a difference in the tone of the color, also: a darker tone indicates more impact.

2. Priority indicators (criticality, budget distribution, float)

Representing priority indicators on the $3 \mathrm{D}$ model can be of great significance. Critical activities of the project decide the duration of the whole project. Any amount of delay in critical activities can delay the whole project. Float is another crucial factor that decides the schedule of the whole project. It shows how much time is left for the non-critical activities to become critical activities. The priority indicators can be represented on the border of the $3 \mathrm{D}$ model. The color scheme can be defined by the user.

\section{Causes of deviation}

Knowing the cause of deviation in the schedule and cost is critical information for project managers. By knowing the cause of deviation, project managers can take proper steps to overcome it. 


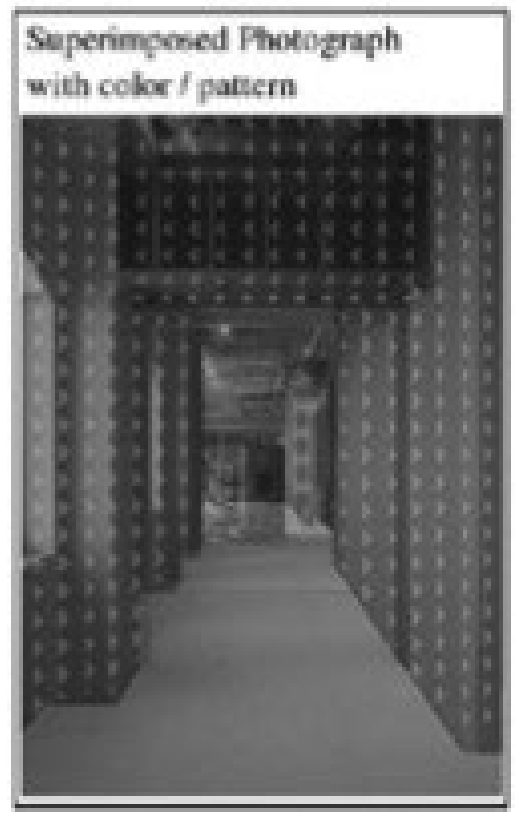

Figure 9 The forward arrow represents the activities ahead of schedule; backward arrow represents those that are behind schedule

(Source: Seungjun et al., 2011)

paper visualizes the project's progress in one holistic view. By this method, all project participants can easily interpret the progress of the project. Also, the construction progress can be monitored remotely. This saves a lot of travelling time of the architects and project executives. This paper presents a model for comprehensively monitoring and detecting schedule deviation in the interior construction work using site photographs and the BIM model. The AR environment allows the as-built and as-planned models to be geo-registered and to visualize the progress by applying a traffic light color pattern. Daily progress photographs are easily available at every project site. These photos can be used in a very effective manner to track the construction progress. Other methods, such as laser scanners, can reconstruct the as-built model very effectively and quickly, but are very expensive and thus cannot be used for smaller, low-budget projects.

\section{Future work}

Research on developing a systematic project progress monitoring system is likely to change the future paradigm of this important project management task. Ongoing research on fully automating the process of monitoring by superimposing the site photographs on the as-planned model is a series of steps in that direction. One of the challenges in the methods discussed in this paper is to monitor the progress of non-physical activities (such as painting or electrical rough-ins). The other challenge is that all the activities in a schedule cannot be represented on the ${ }_{4} \mathrm{D}$ model, and thus, those activities cannot be color coded and tracked. The location of the camera should be properly planned. There are many instances where many of the construction elements (beams, columns) are occluded from other structure; in this case, their progress cannot be monitored. The reconstruction process is very time consuming at times. In an experiment conducted for the Notre Dame Cathedral in Paris, it took 12 days to process and match 2,365 photos and 598 were reconstructed (Snavely et al, 2007). For a large construction project, there are thousands of photos to be processed, for which this approach would not be fully effective. When there are more cameras capturing a single point, the bundle adjustment tends to become slower (Snavely et al., 2007), which in turn slows down the reconstruction process. The process of reconstruction requires extensive manual work. There is ongoing research for speeding up the reconstruction progress. Computer vision techniques for automatically detecting construction elements from the asbuilt photographs and recording the progress have already been developed. Still, the detection techniques need to be improved. The detection of object can be enhanced by photometric properties of the material (color or texture) (Seungjun et al., 2011). The process for interior construction progress monitoring requires more time for preparing a detailed schedule, but the process is more efficient and can save time and money for all project stakeholders.

\section{Acknowledgment}

Contributions of Mr. Parth Shukla, a former graduate student in the University of Maryland Project Management Program, to the above research and to an earlier version of an unpublished report on this subject are hereby acknowledged.

\section{References}

Golparvar-Fard, M., Peña-Mora, F. and Savarese, S. "Monitoring of Construction Performance Using Daily Progress Photo graph logs and 4D As-planned Models." Computung in Civil Engineering. 2009. 53-63.

Golparvar-Fard, M., Peña-Mora, F., Carlos Arboleda, and SangHyun Lee. "Visualization of Construction Progress Monitoring with 4D simualtion Model Overlaid on Time-Lapsed Photographs." ASCE Journal of Computing in Civil Engineering 23 (4) , 2009: 391-404.

Kim, C., Kim, B., and Kim, H. (2013). "4D CAD model updating using image processingbased construction progress monitoring." Automation in Construction, 10.1016/j. autcon.2013.03.005, 44-52.

Kyuman, Song, S. Pollalis, and Feniosky PeñaMora. "Project Dashboard: Concurrent Visual Representation Method of Project Metrics on 3D Building Models." Computing in Civil Engineering (2005). 2005. 1-12.

Lowe, D. "Distinctive image features from scaleinvariant keypoints." International Journal of Computer Vision 60 (2), 2004: 91-110.

Mikołajczyk, K., T. Tuytelaars, C. Schmid, A. Zisserman, J. Matas, F. Schaffalitzky, T. Kadir, L. Van Gool,"A comparison of affine region detectors." Internation Journal of Computer Vision 65 (1/2), 2005: 43-72.

Nister, D. "An efficient solution to the five point relative pose problem." IEEE Transactions on Pattern Analysis and Machine Intelligence 26 (6), 2004: 756-770.

Peña-Mora, Feniosky, Carlos A. Arboleda, Sanghyun Lee, and Mani-Golparvar Fard. "Visualization of Construction Progress Monitoring with 4D Simulation Model 
overlaid on Time-Lapsed Photographs." ASCE Journal of Computing in Civil Engineering, 2009: 391-404.

Roh, Seungjun, Z. Aziz, and F. Peña-Mora. "An object-based $3 \mathrm{D}$ walk-through model for interior construction progress monitoring." Automation in Construction 20 (1), 2011: 66-75.

Snavely, N., S. Seitz, and R. Szeliski. "Modeling the world from internet photo collections." International Journal of Computer Vision, 2007.

Tserng, H., Ho, S., and Jan, S. (2014). "Developing BIM-assisted as-built schedule management system for general contractors." Journal of Civil Engineering and Management, 10.3846/13923730.2013.851112, 47-58.

Turkan, Y., F. Bosche, C.T. Haas, and R. Haas. "Automated progress tracking using $4 \mathrm{D}$ schedule and 3D sensing technologies." Automation in Construction (Volume 22), 2012: 414-421.

Vanlande, Renauld, Christophe Nicole, and Christophe Cruz. "IFC and building lifecycle management 18(1)." Automation in Constructin, 2008: 70-78.

Vanlande, Renauld, Christophe Nicolle, and Christophe Cruz. "IFC and building lifecycle management." Automation in construction (Volume 18 No. 1), 2008.

Wang, Lingyun. "Using 4D Modeling to advance construction visualization in engineering education." May 2007. Penn State Computer Integrated Construction Research Program.

Wikipedia. n.d. http://en.wikipedia.org/wiki/ Industry_Foundation_Classes. 